\title{
Poder, tiempo libre y recreación
}

\author{
Power, free time and recreation
}

\author{
Juan Manuel Carreño Cardozo*
}

\section{Resumen}

\section{Palabras clave: Recreación, poder, tiempo libre, juego, interculturalidad.}

\section{Abstract}

Key words: $\quad$ Recreation, power, free time, play, cross-culturality.

En este artículo se develan las relaciones entre poder, hegemonía, cuerpo y tiempo libre como elementos situacionales de donde parten posibilidades del juego, desde aspectos sociales, comunicativos y educativos, encaminadas a la construcción de un discurso académico que dé cuenta de la recreación como fenómeno social de resistencia y alteridad.

En la primera parte se considera la recreación, en el ámbito sociopolítico, como una manifestación cultural que emerge de un sistema ideológico particular, que posee estructuras de poder demarcadas por la inequidad, la acumulación de bienes y los procesos de legitimación de éste. Dentro de estas estructuras de poder son relevantes las formas en que se internalizan los modelos necesarios para la reproducción del sistema en los sujetos, a través del control del cuerpo y el dominio del tiempo. Posteriormente se presenta el juego desde una perspectiva cultural que por principio se opone al mecanicismo configurado en la tradición occidental, es decir, al activismo recreacionista, a la manipulación de las necesidades lúdicas de la sociedad a través del medio deportivo y turístico, entre otros. Para finalizar, se da cuenta de la recreación como hecho social que contribuye a la conformación de un proyecto de nación propio y a la construcción de tejido social con la disposición de la alteridad como proceso de asunción del otro como otro yo.

This article reveals the relations among power, hegemony, body and free time as situational elements from which different possibilities for play arise, from social, communicative and educational aspects, aimed at constructing an academic discourse that accounts for recreation as a social phenomenon of resistance and alterity.

The first part of the article centers around recreation, in the sociopolitical field, as a cultural manifestation merging from a particular ideological system, with power structures marked by inequity, assets accumulation, and its legitimation processes. Among these structures, the ways in which the models necessary for the reproduction of a system are internalized by subjects, through the control of the body and the domain of time, stand out. In the second part, play is presented from a cultural perspective that, on principle, goes against the mechanicism shaped in Western tradition, that is, against recreational advocacy, manipulation of the ludic needs of society through sports and tourism among others. Finally, recreation is presented as a social phenomenon contributing to the construction of a society's own project of nation, and the social fabric with alterity operating as a process to assume the other as another self.

Fecha de recepción: 10 de mayo de 2006

Fecha de aceptación: 20 de noviembre de 2006

\footnotetext{
* Licenciado en Educación Física (1995), y Especialista en Teorías, Métodos y Técnicas de Investigación Social de la Universidad Pedagógica Nacional (2003). Candidato a magíster en Investigación Social Interdisciplinaria de la Universidad Distrital Francisco José de Caldas (2005). Profesor catedrático de la Universidad Pedagógica Nacional.
} 


\section{Poder y hegemonía}

Sobreentendiendo la dificultad de condensar en pocas palabras la complejidad del problema en que se halla inmerso el mundo desde la perspectiva sociopolítica, se despliegan en forma sintética las principales posturas que refieren la situación, según la mirada analítica de los sistemas ideológicos y de constitución de la soberanía como procesos de construcción histórica. Estos elementos se presentan desde el orden nacional como configuraciones del contexto social de la recreación.

En el ámbito general pueden reconocerse unas formas globales en que se ha construido el poder en el transcurso de la historia de la humanidad, sin ser necesariamente etapas secuenciales, sino alternas o paralelas y presentes en diversos contextos. Primero, la teocracia, donde es Dios quien representa la entidad de poder y son sus designios los que lo legitiman, y aparece la Iglesia como institución comunicadora de su palabra. Segundo, la Ilustración muestra otra forma de poder desde la razón, donde el Estado ha de buscar el bien común a través de acuerdos o contratos. Por último, de la diferenciación en clases sociales deviene una organización del Estado al servicio de una clase, donde es necesario un discurso que favorezca los intereses de ésta. Desde estas formas es visible cómo la constitución del poder en los últimos años no proviene de un pensamiento moderno aislado, sino que hace parte de una construcción histórica donde se entremezclan en un mismo sistema diversas maneras de ejercer el poder.

La hegemonía como un proceso en el cual una sociedad necesita una visión de un Estado fuerte puede generalizarse en las formas en que el gobernante gana legitimidad ante el gobernado. Históricamente, no sólo se trata de un problema de coacción, sino de alianzas, de esta forma se entiende cómo de una legitimación a través de la fuerza de quien ostenta el poder, se pasa a los proyectos educativos donde se sustentan las razones por las cuales una clase debe gobernar. En la educación y los medios de comunicación se presentan en gran medida las alusiones de lo común que sustentan ese poder simbólico legitimador de la hegemonía de un Estado (Bourdieu, 1999).

La soberanía, entendida como mecanismo de poder y relación de fuerzas, se transforma del soberano monopólico individualizado de la premodernidad, a la constitución de sujetos que delegan su derecho de supremacía a otro que lo ejerza en la modernidad (Foucault, 2000). Para Michel Foucault existe una clara diferencia entre el poder jurídico, político y económico, en todo caso "el poder es el poder concreto que todo individuo posee y que, al parecer, cede, total o parcialmente, para constituir un poder, una soberanía política" (Foucault, 2000: 26); para el filósofo francés, el poder es en esencia lo que reprime. Desde esta perspectiva, Foucault propone la hipótesis según la cual el poder es la guerra, y la guerra proseguida por otros medios. La política corresponde a una de esas manifestaciones donde de modo permanente se presenta la situación amigo-enemigo en una confrontación de fuerzas donde es inevitable la relación de subordinación.

Una de las enseñanzas más difundidas de Foucault es la que muestra el poder no en una relación vertical, sino difuminada en la sociedad; y es más importante la forma en que el poder se construye desde abajo. Se pasa, así, de las decisiones soberanas al disciplinamiento de los cuerpos mediante las "técnicas de si". (Foucault, 1976: 40). En síntesis, el autor muestra la manera en que el capitalismo conforma los cuerpos dóciles que necesita, mediante sistemas de dominación, como la medicalización de la sociedad y el biopoder. En estos procesos de dominación es relevante el modo en que el soberano conforma la noción de verdad a partir del imaginario acerca de la ciencia, entonces, las verdades venidas de los científicos están inevitablemente mediadas por las necesidades del grupo dominante. En este sistema de conocimiento, las argumentaciones que han conformado la sensibilidad y los hábitos orgánicos provienen de la misma forma de requerimientos de conductas masivas que favorecen la ideología predominante.

En el contexto actual es necesario entender la soberanía en el ámbito de la constitución de los poderes en potencias (Negri, 2001). Sin necesidad de considerar las posiciones radicales que suponen la globalización como un proceso acabado en que el imperialismo se manifiesta, podemos reconocer fácilmente que la soberanía no sólo se concentra en los Estados-nación, sino que existen otras estructuras de diferentes magnitudes que se desplazan más allá de los límites geográficos, y tienen gran relación con los medios de comunicación y los procesos de consumo.

Veamos, desde estas afirmaciones, algunas de las particularidades que para Colombia enmarcan el hecho social recreativo en un contexto de situaciones muy propias sujetas al entorno mundial; es decir, el colombiano para el cual indagamos manifestaciones lúdicas y proponemos espacios o actividades recreativas es un sujeto atravesado, aunque en formas diversas, por la 
inequidad, el hambre y la velación de la información sobre las condiciones políticas, económicas, educativas y laborales, entre otras.

A la fecha, y según el departamento de estadística del Estado, Colombia tiene un $67 \%$ de su población en el nivel de pobreza, es decir, unos 29 millones de personas subsisten en unas condiciones que se encuentran en el límite de la supervivencia. Así mismo, existen unos siete millones de personas en el nivel de indigencia, cuyo sustento diario no es suficiente para su alimentación ${ }^{11}$. Además, y paralelo al problema mundial, la concentración de riqueza está en manos de muy pocas personas y un solo colombiano puede ganar lo que devengan 72 . Esta inequidad se refleja en los problemas de salud y educación, donde la atención y los recursos difieren demasiado de acuerdo con las posibilidades económicas. Consecuente con la desigualdad en la distribución de las riquezas y la incoherencia administrativa, el desempleo presenta uno de los mayores porcentajes de América Latina e incrementa el subempleo, los oficios informales y el hurto. En un país que incluso ha legitimado las diferencias sociales por medio de los estratos, donde la información es manipulada por los grupos económicos dominantes, la configuración de Nación se construye desde un discurso homogeneizador y diferenciador que la convierte en simple símbolo, instrumento político para predicar ideas sobre identidad y moral frente al otro. Sumado a este panorama se presenta la violencia armada como parte de un conflicto de intereses económicos, políticos y sociales; y paralelo a ello, subsiste la diversidad étnica y cultural y las manifestaciones que le son propias.

En este sentido, las reflexiones y las propuestas acerca de la recreación en Colombia han de dirigirse a un sujeto inmerso en una realidad concreta y a conformar un proyecto de Nación que contribuya al mejoramiento de las condiciones sociales.

\section{El cuerpo construido de la modernidad}

Los procesos mediante los cuales los sistemas ideológicos intentan reproducirse a través del poder y la hegemonía son, para nuestra actualidad, problemas que van más allá de la representación de discursos de gobernantes o funcionarios, y tienden a la internalización de los disciplinamientos del poder en cada individuo (Foucault, 1976; Prieto, 2002). La estructura de dominación, entonces, no parte de una orden explícita, sino

Datos obtenidos del diario La República, lunes 2 de agosto de 2004. de las opciones configuradas en el sujeto por medio del control vital de su existencia: su cuerpo. En éste se concentran los hábitos y las conductas necesarios para la reproducción del sistema e incluso permiten el control sobre el crecimiento y la regulación de las poblaciones (el biopoder). En este punto será comprensible la relevancia del control de los medios de comunicación y la configuración de imaginarios alrededor de la información y los modelos de consumo.

El control de los cuerpos ha sido, históricamente, un elemento clave para la constitución de Estados y la prevalencia de ideologías; sin embargo, desde la teocracia es visible la religión como institución conformadora de represiones y reglas acerca de la conducta. La estructura del proceder religioso no desaparece en la actualidad, sino que se mezcla en el sentido común de forma desordenada pero con una disposición rigurosa en el comportamiento. Es importante mencionar la forma en que el discurso civilizador de la Edad Media en el descubrimiento de América contiene el elemento religioso como forma de diferenciar lo salvaje de lo divino, como elemento conformador de las jerarquías necesarias para la dominación (Pedraza, 1999). La religión ha presentado, en términos generales, el medio de represión de los deseos por excelencia, conjugando el miedo al castigo infernal con las necesidades de trascendencia vital.

La medicina también evoluciona en forma evidente desde la disección de los cadáveres, y transforma, a través de sus descubrimientos, los discursos sobre salud, y los imaginarios y las costumbres de aseo, alimentación, salud, etc. El discurso médico positivo se transforma en una "gramática de los signos" (Martínez, 2003: 14), donde el cuerpo se fragmenta en el más mínimo órgano y parte, y conforma una sensibilización del sujeto hacia su organismo, de manera análoga al cuidado de una máquina, de la cual se estima sólo su funcionamiento y su duración. La medicina divide aún más el cuerpo dual, y lo segmenta para ser sentido de esa manera. Así, el poder predominante se encarga de representar desde la salud y la enfermedad, modelos a seguir, inhibiciones, represiones, y a separar definitivamente en el imaginario del sujeto el aspecto humano de las necesidades orgánicas.

Aparte de la religión y la medicalización como aparatos transversales en la constitución y el dominio del imaginario actual de cuerpo, el sujeto moderno se conforma por otros muchos elementos que, de manera cada vez más compleja, se conjugan en los sistemas de poder como mecanismos de dominación. En esta forma se reconoce en la actualidad un cuerpo objeto 
de intercambio en los procesos de consumo (Bernard, 1985; Martínez, 2003; Viveros y Garay, 1999), donde el cuerpo es, por un lado, un patrón de representación homogenizador y una mercancía, y por otro, un cuerpo consumidor: el sujeto que trabaja para la adquisición de bienes y el acercamiento a los modelos impuestos por la sociedad. Los cambios en los sistemas laborales son, precisamente, otro elemento relevante en la mecanización del cuerpo, donde el sujeto es medido de acuerdo con su capacidad de producción y, así mismo, se educa y se desarrolla como una máquina de producción: su cuerpo es herramienta de trabajo y es sometida al rendimiento. Serán necesarios, también, mecanismos para establecer un descanso y una diversión moderados que, en la mejor de las visiones de Aldous Huxley, constituyen el espacio de felicidad del sujeto y el objetivo de la existencia.

Esta construcción del cuerpo moderno se estructura mediante los mecanismos de información y entretenimiento, dirigiendo la sensibilidad común de una sociedad hacia la creación de nuevas necesidades y la manipulación de las expectativas hacia posibilidades limitadas. También se encarga de individualizar los problemas, haciendo responsable de las deficiencias a cada sujeto y a su actitud ante la vida, y desdibujando el papel del Estado ante los problemas. Las percepciones del mundo se controlan, no desde las conciencias, sino desde la carne: el sujeto siente en forma tangible esas necesidades de consumo y representación. También construye los procesos de exclusión, desde los imaginarios cimentados en los patrones, es decir, el seguimiento de modelos constituye al sujeto como el siempre incluido, y la visión de otro, como el excluido de las posibilidades de desarrollo que aquel incluido sí posee. En este proceso los sistemas de dominación hacen imperante la concientización del sujeto como parte de un mundo que está a su alcance, mediante el supuesto acceso ilimitado a la información y la integración de la conciencia colectiva en la noción de opinión pública (Chomsky, 1995).

En este sistema homogeneizador, conformador de cuerpos dóciles al servicio de la producción y el consumo, siempre existen manifestaciones de desacuerdo y posibilidades de salida a los procesos de dominación. Éstos surgen por la variabilidad del ser humano y la dinámica de su comportamiento no lineal, por la imposibilidad de la homogeneización total, la magnitud de las poblaciones, la carencia de mecanismos más eficaces de masificación, entre otros. El sujeto, conformado por la represión de sus deseos y el disciplinamiento de la norma a través de su cuerpo, busca de modo consciente o inconsciente salidas, oportunidades que dan cuenta de su visión del mundo desde sus sentimientos y reflejan la pasión que no alcanzan a controlar los sistemas de dominación.

La opción de la recreación es constituirse en discurso legitimador del poder dominante o en el intento de subvertir el orden establecido. Desde esta perspectiva se desdibujan los supuestos malos entendidos de las nociones de juego y recreación y se da la posibilidad de aplicar a los diversos entendimientos y usos la tesis planteada: las propuestas, los discursos y los proyectos se disponen como reproductores del sistema o como formas resistentes a él. En ambos casos, la recreación retoma esas manifestaciones inconformes y diversas desde la sensibilidad del sujeto, desde la construcción de sentidos y significaciones cotidianas acerca del mundo, en un caso para distraer de la situación, y en el otro para darse cuenta de ella.

\section{Tiempo laboral frente a tiempo ocioso}

El sujeto de la recreación, como lo hemos descrito, se encuentra situado en un contexto específico según unas estructuras de poder y hegemonía construidas históricamente en su cuerpo como objeto de relación con un mundo productivo, también como objeto de deseos, necesidades y expresiones. Así mismo, el cuerpo se encuentra ubicado en un espacio y un tiempo determinados, que despliegan los límites en los cuales el ser humano actúa en el mundo, por tanto, también determinan su configuración de mundo. En los discursos sobre recreación, el tiempo libre es un núcleo obligatorio de análisis, de los cuäles retomaremos las ideas más destacadas, para asentar las relaciones entre el control del tiempo y el hecho recreativo desde la perspectiva social del poder.

Cuando se plantea una referencia al tiempo libre, debe entenderse el punto de vista desde el cual parten las disertaciones que se han dado sobre éste. Un lugar de partida que al parecer ha sido común a estas discusiones es la diferenciación inicial entre el tiempo de trabajo y el tiempo que está fuera de éste. En principio, el tiempo de trabajo puede delimitarse fácilmente como el tiempo que el individuo destina a actividades productivas, remuneradas y dependientes de su carácter económico (Munné, 1980). El tiempo libre se enmarca, desde el referente anterior, en el tiempo que está fuera del tiempo de trabajo; sin embargo, en éste se presentan actividades obligatorias como las tareas domésticas, los compromisos sociales, educativos, entre otros (Rodríguez, 1996; Ortegón, 1998; Rodríguez, 1992). Aunque parecen po- 
los opuestos, el tiempo de trabajo y el tiempo libre no se encuentran distanciados en una linealidad, sino que tal oposición pertenece a una interpretación determinada de nuestra realidad (Munné, 1980), que enmarca las valoraciones desde lo productivo (económico) y lo improductivo del tiempo del sujeto. A partir de la diferenciación del tiempo libre en los aspectos que son de igual modo imperiosos para su subsistencia, se delimita un tiempo en el cual el individuo puede decidir libremente su actividad, la dirige en forma autónoma y es considerada como satisfactoria y gratificante en el marco de un crecimiento personal. A este tiempo se le ha denominado tiempo liberado (Cuenca, 1998). El ocio, como posibilidad dada por el tiempo liberado, es sinónimo de "ocupación gustosa, querida y (...) libremente elegida" (Cuenca, 1998: 6), atravesada por los significados que contiene la experiencia individual de su emotividad y, consecuente con ello, de su felicidad.

Surge, entonces, el problema de la libertad como estructura informe, que se transforma y, para nuestro caso, es manipulable a través de las formas en que una sociedad determina las elecciones del individuo y es capaz de fomentar un imaginario etéreo de libertad, sobre el cual se elaboran los espacios de consumo. La discusión se ubica en comprender hasta qué punto la libertad del individuo se determina o no por estructuras sociales masivas.

De acuerdo con la posición kantiana según la cual "el hombre es libre porque es un sujeto moral dotado de razón" (Cioffi, 2003: 2), gran parte del pensamiento moral moderno concibe la libertad, de acuerdo con lo dicho sobre el ocio, como autorrealización, expresión y expansión del yo. La diferencia radica en la consideración de la libertad desde un solo punto de vista ético aislado y asumirlo en el ámbito de relación con los otros. Éste refiere a la libertad política y social, en cuanto el individuo se constituye como titular de derechos involucrado en un conjunto de normas y símbolos que determinan sus opciones. Las formas democráticas y liberales de Estado pueden conformar, a través de la uniformidad, la masificación y el conformismo, las brechas más grandes entre éstas y la libertad; es la tiranía de la mayoría puesta al servicio del sistema dominante (Cioffi, 2003). En sintesis, podemos definir el problema del tiempo libre en relación con el poder, como la necesidad de establecer la noción de libertad en el contexto actual, donde existe la posibilidad de pensamiento de libertades individuales confrontadas con la libertad social determinada en gran medida por una ideología dominante y controladora de pensamientos, creadora de nuevas necesidades y mani- puladora de deseos a partir de las figuras de la democracia, la participación y la misma libertad.

En forma tangible podemos determinar que la tendencia del sistema no es la pretensión de un individuo con gran capacidad de elección. Es decir, a todas luces persiste el control de dichas libertades, no en la prohibición, sino en la limitación de las opciones interiorizadas de modo inconsciente. Los medios de comunicación actúan según las estructuras del mercado y garantizan su existencia por la manipulación de la capacidad de consumo del individuo. La masificación da cabida a la ley del "menor esfuerzo" y desdibuja oportunidades de acción frente a las opciones de diversión mercantilizada. La felicidad se presenta como la consecución del tiempo destinado a lo que los medios de comunicación proponen y venden, programada para cada sujeto y cada contexto en forma precisa con sus patrones a seguir.

La recreación se incluye de modo inevitable en el tiempo liberado del individuo; también se puede disponer en una actitud diversa en las relaciones de obligación y descanso. Esta disposición ha de retomar la libertad como oportunidad de florecimiento de las particularidades de los individuos a favor de una sociedad articulada desde el diálogo, y no desde la homogeneización. La posibilidad de la recreación es la ampliación de los límites sociales durante el tiempo liberado, la opción de sensación y resignificación de los sentidos de trascendencia y felicidad del individuo.

\section{El juego: posibilidad de restauración desde la interculturalidad}

El juego es un fenómeno social del cual se han elaborado diversos discursos que pretenden su definición y su teorización. Para la presente reflexión, nos abstendremos de entrar en discusiones profundas sobre su término y sus límites, ya que, en principio, el juego se constituye en forma diversa en las culturas, es también construcción social que depende de una elaboración histórica importante. En forma general, retomaremos los elementos de la propuesta de Huizinga (1972) que relacionan el juego con la cultura, desarrollando su principio lúdico como fundamento de la relación entre éste y la situación de poder que aquí nos interesa. El juego es, entonces, manifestación lúdica de una cultura y constituyente de la misma. Para nuestro contexto occidentalizado, podemos generalizar que del juego se subvalora su carácter creativo y se le relega, en el momento en que se asume el proyecto de la razón, desde la cual la lógica normativiza 
la visión del mundo y se asume el capital y sus sistemas de producción

En gran parte de los discursos sobre el juego se valora su principio creador e imaginativo (Toro, 1996; Bonilla, 1997). Esto constituye el nuevo paso hacia el reconocimiento del juego con un valor que desdibuja su medición desde la utilidad productiva. Sin embargo, una es la situación de los discursos y otra muy diferente la de los sentidos que aparecen en lo cotidiano. Es decir, el juego en el imaginario social continúa siendo relegado a una actividad encerrada en la edad infantil, y cuando mucho, se estructura en procesos educativos que propendan por una formación intelectual más eficiente. El cuerpo del sujeto que tiene necesidades lúdicas queda privado de una posible manifestación en el juego.

Estas necesidades visibles se vierten hacia la elaboración de instrumentos que canalicen la energía de una sociedad en su tiempo liberado; aparece el deporte como una herramienta esencial en la dominación del tiempo de la sociedad. Sin entrar en una discusión moral acerca del deporte, sólo nombraremos algunos elementos que contienen lo necesario para la reproducción de una ideología homogeneizadora y hegemónica: la formación de la estructura de competencia y de desconfianza, la propuesta de modelos comercializables fácilmente, la constitución de opciones ya determinadas sin oportunidad de cambios, la perspectiva de continua comparación y subordinación por los resultados, etc. Aunque no se trata sólo del deporte, éste sí es un ejemplo visible de la estructura de dominación junto con las formas de la recreación activista, manifestada en la facilitación de espacios de mecanización del descanso.

El juego en nuestro contexto tiene posibilidades de elaboración discursiva amplia, que van más allá de las limitaciones que plantea el imaginario global de Occidente, desde la facilitación de una perspectiva intercultural, que, por principio, debe reconfigurar el orden jerárquico donde el saber tradicional se ha relegado. En este sentido, resulta imperante el reconocimiento del espacio nacional como campo de interlocución que posibilite la discusión y el intercambio de saberes, conocimientos o representaciones para la construcción o la reformulación de otros nuevos. Por ejemplo, se podría pensar que la representación social del juego como fenómeno cultural, junto con su carácter lúdico, difiere dependiendo del contexto; desde allí se originan nuevas perspectivas frente al juego y la recreación.

En nuestro país es posible restaurar un espíritu lúdico encaminado a propuestas pedagógicas, sociales o artísticas, que tiendan a la construcción de un proyecto coherente de nación, desde la comprensión del juego como fenómeno fundamental de las diversas culturas que habitan nuestra geografía.

\section{Conclusión: la recreación como fenómeno social de resistencia y alteridad}

La recreación en nuestro país presenta un proceso dis continuo y de múltiples intentos de acercamiento a propuestas globales, donde se evidencian incoherencias administrativas, desorden en la inversión y tentativas de asimilación de procesos observados en otros países. La recreación se conforma en el contexto colombiano como una posibilidad acorde con la capacidad económica individual, donde el discurso jurídico y político garantiza el acceso, pero no se concretan condiciones que lo permitan (Garzón et al., 2004). Paralelo a esto, se priorizan la actividad deportiva y el espectáculo, dentro de la confusión en la indistinta utilización de los términos recreación, deporte, juego, educación, entre otros, aplicados a proyectos cuyo propósito no se concreta.

Como respuesta académica surgen diversas inves tigaciones y propuestas que permiten establecer bases para una discusión acerca de la recreación en nuestro contexto. Estas propuestas se desarrollan desde diversos campos, como la Sociología, la Comunicación, la Educación Física, la Administración, la Antropología, etc. Sin embargo, puede observarse que la mayor parte de los trabajos en este campo se inclinan a estudiar y proponer desde la lúdica, entendida como una actitud y una necesidad del ser humano de sentir, expresar, comunicar y producir emociones (Bonilla, 1997), tomando de modo tangencial la recreación como posibilidad de re-crear, pero sin ahondar en sus potencialidades como hecho social que permite organizar y proyectar esfuerzos hacia la constitución de un proyecto de $\mathrm{Na}$ ción desde sujetos críticos, re-pensadores y re-creadores de su realidad.

Para encaminar el tipo de propuestas mencionadas es necesario interpretar las relaciones existentes entre esos aspectos, aparentemente inscritos en la teorización, y la situación sociopolítica, histórica y cultural, tanto en el plano general como de casos particulares; de manera que los discursos se vinculen, desde sus fundamentos, con las reflexiones acerca de las estructuras de poder y los disciplinamientos que inevitablemente interactúan con fuerza en la actualidad. Es decir, establecer de modo decidido la perspectiva de estudio de la recreación asociada en forma directa con el problema del poder, el 
control y atravesada de modo inevitable por situaciones sensibles que particularizan el saber sobre recreación en América Latina. Esto se concreta haciendo manifiesta el hambre, la inequidad, la violencia y otros hechos arraigados en el contexto.

Como se ha evidenciado en este escrito, la presente reflexión toma partido por la visión del hecho recreativo como proceso de resistencia ante los aparatos de masificación y dominación. Por una parte, interpretando éste como una manifestación colectiva de inconformidad hacia el sistema, y, por otra, dando cuenta de la responsabilidad ética de promover la conciencia crítica ante una situación de pobreza e inequidad. Es aquí donde el proceso educativo puede reconfigurarse más allá de las disciplinas, al dar oportunidades de formación de licenciados con capacidad de interpretación del contexto, y con habilidad profesional para promover el espíritu lúdico liberador en la educación, ya sea formal o informal (Garzón et. al., 2004).

En este mismo proceso de formación, la recreación puede facilitar procesos de alteridad, entendida ésta como la posibilidad de asunción del otro como otro yo. En este sentido, permite que el sujeto reflexione no sólo sobre su situación sino sobre la situación de los que están con él. Este conjunto de diálogos hace que los saberes se constituyan en el tejido sobre el que reposen los imaginarios y en lugar de estructuración de propósitos colectivos.

\section{Referencias}

Bernard, M. (1985). El cuerpo. Barcelona: Paidós.

Bonilla, C. B. (1997). Una aproximación al concepto de lúdica. Kinesis, 22.

Bourdieu, P. (1999). ¿Qué significa hablar? Madrid: Akal.

Chomsky, N. (1995). Cómo nos venden la moto. Barcelona: Icaria.

Cioffi, F. (2003). ¿Cuándo somos libres? Bogotá: Universidad Pedagógica Nacional.

Cuenca, M. (1998). Significado actual de ocio. Cuadernos de ocio, 2. Foucault, M. (1976). Vigilar y castigar. Bogotá: Siglo XX. . (2000). Defender la sociedad. Buenos Aires: Fondo de Cultura Económica.

Garzón, L. A. et al. (2004). Programa académico de Licenciatura en Recreación (documento de circulación interna, inédito). Bogotá: Universidad Pedagógica Nacional.

Huizinga, J. (1972). Homo Ludens. Madrid: Alianza.

La República, lunes 2 de agosto de 2004.

Martínez, A. (2003). El cuerpo imaginado de la modernidad. Debates, 79 .

Munné, F. (1980). Psicosociología del tiempo libre. México: Trillas.

Negri, A. (2001). Soberanía. American Anthropological Association: Washington.

Ortegón, R. (1998). Educación para el tiempo libre. Bogotá: Asociación Colombiana de Recreación.

Pedraza, Z. (1999). En cuerpo y alma. Bogotá: Universidad de los Andes.

Prieto, H. (2002). Resistir a la administración total de la vida. Bogotá: Universidad de El Bosque.

Rodríguez, J. (1992). Tiempo y ocio. Bogotá: Universidad Externado de Colombia.

Rodríguez, M. (1996). Del tiempo libre a la recreación. Educación Física, 240.

Toro, G. (1996). El juego: nacimiento de su permanente presencia. Educación Física y Deporte, 8 (1).

Viveros, M. y Garay, G. (1999). Cuerpo, diferencias y desigualdades. - Bogotá: Universidad Nacional de Colombia, Facultad de Ciencias Humanas. 